\title{
La responsabilidad social empresarial: del cumplimiento de la ley a la construcción de un modelo de gestión y una filosofía empresarial y organizacional
}

\section{Julianna Paola Ramírez Lozano}

\section{Resumen}

La responsabilidad social va dejando de ser un tema de interés solamente para las empresas privadas, pues lo es también para las organizaciones públicas y del tercer sector; cualquiera sea su tamaño, ubicación y objetivos, están llamadas a ser socialmente responsables. Sin embargo, la responsabilidad social en el Perú aún es un término que no ha logrado conocerse y aplicarse por completo en las organizaciones, a pesar del interés manifestado por muchas de ellas.

La responsabilidad social debe entenderse como un modelo de actuar, que las organizaciones deciden adoptar de manera voluntaria y siguiendo un proceso de incorporación de políticas, acciones y estrategias de responsabilidad social. Esto tiene un impacto no solo en los grupos de interés, o stakeholders, que se relacionan con la organización, sino también para la propia empresa y organización.

La responsabilidad social se debe plasmar en una serie de programas, proyectos y prácticas en beneficio de los diversos grupos de interés, los cuales tienen como punto de partida el cumplimiento de la ley. Sin embargo, la responsabilidad social empresarial incentiva a las empresas a desarrollar acciones que vayan más allá de lo exigido por la ley. Estas 
acciones nacen de la iniciativa y necesidad de las organizaciones y empresas, por su afán de mantener buenas relaciones con su entorno.

Los proyectos sociales dirigidos hacia la comunidad resultan ser una manera visible y concreta desde la cual la empresa u organización puede manifestar su ayuda a la mejora de la calidad de vida de su entorno, lo cual es parte de su responsabilidad social. Para explicar las variables de impacto de los proyectos sociales, presentaremos el caso de tres organizaciones que trabajan en el Perú1: Petroperú, empresa pública, BBVA-Continental, empresa privada, y Cáritas del Perú, organización del tercer sector. Estos casos nos muestran cómo estas organizaciones, de diferente gestión económica, pueden desarrollar acciones de responsabilidad social de gran impacto en la sociedad peruana, que a su vez generan un impacto positivo importante en la imagen y reputación corporativa de las mismas organizaciones.

\section{Introducción}

El punto de inicio para que las empresas privadas, públicas y del tercer sector puedan convertirse en organizaciones socialmente responsables lo encontramos en el enfoque de su misión, visión, principios y valores de la propia organización. Es decir, la responsabilidad social debe entenderse desde el interior, como una filosofía, como una manifestación de hacer bien las cosas y de retribuir al entorno los beneficios obtenidos como producto de la venta o comercialización de productos o servicios.

En el Perú, el concepto de responsabilidad social empresarial no es del todo entendido e interpretado. Para algunas empresas es tan solo una manera de maquillar el ejercicio diario, y otras lo conciben más bien como una acción de marketing en beneficio de la imagen corporativa de la empresa, realizando acciones para el bien de uno de sus stakeholders, como las comunidades. Sin embargo, la primera responsabilidad social de las empresas y organizaciones está dentro de ellas mismas, en la llamada responsabilidad social interna, que no es otra cosa que velar

1 La presente comunicación relata los resultados de la investigación realizada por la autora de este texto, gracias a los fondos del Instituto de Investigación Científica de la Universidad de Lima. La investigación se llevó a cabo entre abril del 2013 y marzo del 2014. Trabajaron en ella, en calidad de asistente, Andrés Vernal Peñaranda, y como alumno practicante, Ever Luna Choque. 
por la buena condición de los colaboradores de la empresa; una acción que va más allá de lo exigido por la ley. Sin duda, la adecuada gestión de la responsabilidad social interna debería ser la primera y principal responsabilidad de toda organización.

Como bien menciona el management moderno, la responsabilidad social de la empresa debe ser entendida como un modelo de gestión. En este sentido, el ejercicio de la responsabilidad social pasa por el conocimiento y reconocimiento de los grupos de interés o stakeholders. Por tanto, resulta incoherente que la empresa desarrolle acciones hacia sus stakeholders externos sin antes haber trabajado una razonable acción de responsabilidad social interna. Por esto se dice que las empresas deben "partir por casa", es decir, implementar una política de responsabilidad social con su propio capital humano. Por tanto, la responsabilidad social empresarial se convierte, en el ámbito interno, en un elemento cohesionador, motivador y propiciador del buen clima laboral, lo que lleva en el corto plazo a una mayor productividad y compromiso por parte de todos los colaboradores. Sin embargo, el proceso de incorporación de prácticas de responsabilidad social pasa por la creación de diversas acciones con cada grupo de interés. No existe una receta única para la creación de acciones de responsabilidad social. Lo importante es tener un comportamiento coherente, ético y de respeto por los grupos de interés. La responsabilidad no necesariamente son acciones filantrópicas, sino el respeto de la ley y la apertura a nuevas acciones que traigan consigo un beneficio compartido entre la empresa y sus grupos de interés.

\section{Metodología de la investigación}

Nuestra investigación partió de una revisión bibliográfica acerca de la manera como era concebida la responsabilidad social en las organizaciones públicas, privadas y del tercer sector. Posteriormente, se realizó un trabajo de campo mediante el uso de técnicas de investigación cualitativas, en las tres empresas antes mencionadas:

a. Entrevistas a los principales directivos y gerentes de las tres empresas estudiadas. En total, se realizaron más de 32 entrevistas con profundidad (ver anexos 1,2 y 3 ).

b. Trabajo de campo: consistió en la visita al proyecto más importante de cada organización, lo que incluyó la realización de entrevistas con 
profundidad a los actores más importantes de la gestión del proyecto y a sus beneficiarios directos. En el caso del proyecto "Adopción del Colegio Politécnico Alejandro Taboada", de la empresa Petroperú, se viajó a la refinería de Talara, Piura (anexo 4). El segundo proyecto visitado fue el colegio "Javier Pérez de Cuéllar", como institución educativa emblemática del programa "Leer es estar adelante", de la Fundación del BBVA-Continental (anexo 5). Finalmente, se viajó a la región de Ancash para conocer el programa social "Ally Micuy" de Cáritas del Perú, financiado por la empresa minera Antamina (anexo 6). En total, se desarrollaron 48 entrevistas con profundidad.

\section{La responsabilidad social en las organizaciones peruanas públicas, privadas y del tercer sector}

La responsabilidad social puede ser entendida como una manera de actuar de las organizaciones, independientemente de su gestión económica y del rol que desempeñen en la sociedad. Es decir, la responsabilidad social puede ser aplicada a cualquier tipo de organización -pública, privada o del tercer sector-, sin importar su tamaño, la propiedad, la ubicación o el rubro. Todas las organizaciones, al igual que las personas sobre la tierra, tienen una responsabilidad social con su entorno.

La responsabilidad social debe ser entendida como un compromiso social que asume la organización de manera voluntaria y como manifestación de su interés por contribuir con el bienestar de su entorno. Es un conjunto de acciones que van más allá de las exigencias de la ley y que deben plasmarse dentro de tres componentes del nuevo triple balance, es decir, ser socialmente responsable en el ámbito social o en relación con las personas vinculadas directa o indirectamente con la empresa, ser socialmente responsable con el medio ambiental y cumplir con un buen gobierno corporativo basado en la ética ${ }^{2}$.

Matilde Schwalb y Oscar Malca, estudiosos peruanos del tema, describen la responsabilidad social de la siguiente manera:

La responsabilidad social es una filosofía, una actitud o forma de ver la vida que implica que tomemos en cuenta el efecto de nues-

2 El gobierno corporativo hace referencia al comportamiento ético de las personas que gobiernan o toman las decisiones de la empresa u organización. El gobierno corporativo parte de la ética de las personas. 
tras acciones y decisiones sobre el entorno físico y social. En otras palabras, ser socialmente responsable significa ser consciente del daño que nuestros actos pueden ocasionar a cualquier individuo o grupo social (Schwalb \& Malca, 2005, p. 103).

Una de las premisas de la responsabilidad social es la transparencia, lo cual se evidencia en la necesidad que tienen las organizaciones de evaluar y reportar sus acciones realizadas mostrando resultados. Para esto, las organizaciones necesitan comunicar a todos sus grupos de interés lo realizado en materia de responsabilidad social, dentro de un periodo puntual que puede ser de uno o dos años. Esta labor se realiza mediante un documento llamado reporte de sostenibilidad o balance social. Para su desarrollo se toman en cuentan diversos indicadores; entre los más importantes están: el autodiagnóstico ETHOS, el Global Reporting Iniciative o GRI, el Pacto Mundial, los Objetivos del Milenio y la ISO 26000.

\section{La responsabilidad social en las organizaciones públicas, privadas y del tercer sector}

\subsection{La responsabilidad social en las organizaciones públicas}

El sector público es el conjunto de organismos administrativos mediante los cuales el Estado cumple, o hace cumplir, la política o voluntad expresada en las leyes del país. Esta definición funciona igual para todos los países, pues las organizaciones públicas se rigen bajo la misma clasificación. El Estado está dividido en tres poderes, además de otros organismos: el poder legislativo, el poder ejecutivo, el poder judicial, y otras instituciones públicas autónomas, empresas y personas que realizan alguna actividad económica en nombre del Estado.

Estos organismos representan al Estado en sus diferentes áreas y deben hacerse responsables de sus acciones, que son todas aquellas actividades que el Estado (administración local y central) posee o controla. El papel y el volumen del sector público dependen en gran parte de lo que en cada momento se considere de interés público.

Fernando Navarro reflexiona acerca de las responsabilidades y desafíos que tiene el Estado:

Existen funciones públicas que nadie sino el Estado puede realizar con eficacia [...] una actividad pública es para siempre, no puede liquidarse, venderse o disolverse como una empresa. De la misma 
manera que la actividad pública raramente abandona sus funciones. Una actividad pública tiene más de una misión [...]. (2008, p. 147)

La historia de la responsabilidad social en el sector público no tiene un inicio fijo, pues los organismos públicos nacieron con la razón de servir a la población. Hay documentos que datan del siglo XIX que dan fe de las acciones por parte de algunas compañías públicas en favor de sus trabajadores. Por ejemplo, en ciertas empresas públicas europeas se dieron cuenta de que si empezaban a hacer acciones favorables dirigidas a los trabajadores de la empresa, estos laborarían mejor y las relaciones con el entorno progresarían.

La responsabilidad social para las organizaciones públicas se plantea como el punto de partida desde el cual debe replantearse el equilibrio entre el desarrollo económico y el desarrollo social necesario para construir el nuevo tipo de sociedad, sobre todo en la actualidad, cuando, como bien sabemos, existen serios problemas sociales, sobre todo en algunas partes del mundo, como es el caso de América Latina.

\subsection{La responsabilidad social en las empresas privadas o con fines de lucro}

Diversos autores señalan que la responsabilidad social en las empresas se remonta al siglo pasado. Hay algunos hechos históricos e iniciativas que han propiciado el origen del concepto de responsabilidad social, entre ellos la Conferencia de Estocolmo en 1972, el Informe Brundtland en 1987 y el protocolo de Kyoto en 1997; el nacimiento de grupos civiles como Amnistía Internacional, WWF, Greenpeace o Transparencia Internacional; así como iniciativas formales empresariales como AA1000, SA8000, ISO 26000, las Directrices de la OCDE, el Pacto Mundial; así como el surgimiento de organizaciones como la Global Reporting Initiative (GRI) y el Instituto Ethos, entre otros.

Existen cinco áreas o temas importantes en los que se basa el ejercicio de la responsabilidad social de una empresa, en relación con sus grupos de interés: respeto a los principios éticos y valores, condiciones y el ambiente de trabajo dirigido a los colaboradores o trabajadores, el apoyo a la comunidad cercana donde opera la empresa u organización, la protección del medio ambiente durante la producción o comercialización de los productos o servicios, y finalmente un marketing responsable acerca de la comercialización de los productos o servicios, dirigido a los clientes o consumidores. 
Los objetivos que llevan a una empresa privada a desempeñar un modelo de gestión socialmente responsable se basan en la adopción de una filosofía desde la cual se realice el negocio en su día a día. Es decir, las acciones de responsabilidad social para la empresa privada van ligadas directamente a la necesidad de hacer bien las cosas, de velar por un buen comportamiento en relación con los grupos de interés, basado siempre en la ética, la transparencia, la sostenibilidad, el largo plazo y el "ganar-ganar" de los grupos de interés y de la empresa.

\subsection{La responsabilidad social en las organizaciones del tercer sector}

La existencia de organizaciones del tercer sector no es un fenómeno reciente. Desde el siglo XIX han existido distintas organizaciones sin fines de lucro y más bien con fines sociales. Una de las más antiguas y conocidas es la Cruz Roja. El término fue utilizado por primera vez en 1973, en un artículo de Theodore Levitt cuyo título es "The Third Sector: New Tactics for a Responsive Society", en el que se describe al tercer sector como el espacio de actuación pública que no cubren ni el Estado ni el mercado.

El tercer sector desarrolla actividades voluntarias y altruistas realizadas por diversas ONG, que cumplen un rol importante en la sociedad debido a que surgen del entorno privado de la ciudadanía, generalmente a cargo de activistas sociales que buscan trabajar de manera desinteresada en diversos temas dirigidos a terceros, es decir, en la esfera pública. García Delgado define el tercer sector de la siguiente manera:

El resultado de la capacidad creativa de la sociedad para satisfacer nuevas necesidades y atender las crecidas expectativas que despierta el desarrollo económico y el cambio social en las sociedades libres [...] lo que no pertenece al ámbito de la hacienda pública ni lo que es propio de la actividad mercantil lucrativa, sino como afirmación de una doble correlación. Por una parte, la que en el plano de los objetivos o fines perseguidos se establece entre interés colectivo o general y solidaridad; por otra, la que en el plano de lo organizativo enlaza participación con responsabilidad. (2004, p. 20)

En la actualidad, las organizaciones del tercer sector han sufrido un acelerado proceso de maduración, con un importante aumento de sus actividades y una creciente complejidad organizativa. Las ONG hoy en día son organizaciones que tienen interacciones continuas con su entorno social, las cuales desde ya están enmarcadas en acciones socialmente 
responsables. Se puede establecer que el objetivo máximo que caracteriza y define a las organizaciones del tercer sector es la búsqueda del bienestar y mejora de la calidad de vida de los públicos a quienes se dirigen. Como dice la autora Salas, "se busca la realización plena de las capacidades humanas y el acompañamiento en la búsqueda de la felicidad individual y colectiva" (2004, p. 19). En este sentido, la gestión de la responsabilidad social en las organizaciones del tercer sector resulta ser un acto inherente a su razón de ser.

\section{Perfil de las empresas analizadas: Petroperú, BBVA -Continental y Cáritas del Perú}

\subsection{Petróleos del Perú (Petroperú)}

Fue creada el 24 de julio de 1969, durante el gobierno del presidente Juan Francisco Velasco Alvarado. Petroperú es una empresa estatal peruana y de derecho privado, dedicada al transporte, la refinación, distribución y comercialización de combustibles y otros productos derivados del petróleo. Petroperú emplea alrededor de 2500 personas, entre hombres y mujeres.

La empresa realiza importantes inversiones en educación, salud y otros proyectos de desarrollo sostenible (Petroperú, 2012). Petroperú manifiesta que su desarrollo sostenible y su responsabilidad social se basan en cinco pilares: social, ambiental, económico, cultural y ética de producción; desde los cuales pone en práctica sus acciones.

La misión de Petroperú es satisfacer las necesidades del mercado de hidrocarburos con productos de calidad internacional y servicios competitivos, con adecuada rentabilidad, que propicia el desarrollo de sus trabajadores, y actúa con responsabilidad social y ambiental. Mientras que la visión de la empresa es ser una empresa de hidrocarburos y energía, integrada y competitiva, líder en el mercado nacional y con participación creciente en el mercado internacional.

\subsection{Banco BBVA-Continental}

Es una entidad financiera en los ámbitos nacional e internacional, subsidiaria de Holding Continental S.A., empresa peruana formada por capital peruano del grupo Brescia (50 \%) y capital español del grupo BBVA (50\%). El banco desarrolla sus actividades a través de una red nacional conformada por 266 oficinas distribuidas en el país y centrali- 
zada en su sede principal, ubicada en la ciudad de Lima, capital del Perú (BBVA-Continental, 2012).

BBVA-Continental describe su misión (BBVA-Continental, 2012, p. 12) de la siguiente manera: aportar las mejores soluciones a nuestros clientes, un crecimiento rentable a nuestros accionistas y progreso en las sociedades en las que estamos presentes. Su visión es: trabajamos por un futuro mejor para las personas. Con este concepto transmite su perspectiva como empresa y engloba su principal objetivo en el lado humano: las personas.

\subsection{ONG Cáritas del Perú}

Es un organismo de la Iglesia católica, con 55 años de vida institucional. $\mathrm{Su}$ fin primordial consiste en fomentar iniciativas para que las personas en situación de pobreza y exclusión fortalezcan sus capacidades y oportunidades para mejorar sus condiciones y calidad de vida, bajo el enfoque del desarrollo humano integral, basado en la doctrina social de la Iglesia católica (Cáritas, 2012).

Cáritas del Perú define su misión de la siguiente manera (Cáritas, 2012, p. 15): es el servicio de la caridad y la solidaridad que es ley de vida del Reino de Dios, que va madurando aquí y ahora en la historia hacia su plenitud, animando, acompañando y comprometiéndose, a la luz del Evangelio y desde la doctrina social de la Iglesia, en el proceso de transformación de la sociedad peruana y por el bien común, viviendo como auténticos discípulos misioneros de Jesucristo, creando conciencia social e impulsando procesos de promoción integral de los pobres y excluidos para que asuman un liderazgo cristiano al servicio de sus comunidades; defendiendo el don de la vida desde su concepción hasta su término natural, la familia y la creación, promoviendo el uso racional de los recursos naturales en los procesos de desarrollo sostenible. Mientras que su visión es: para el año 2020, ser una sólida red nacional católica promotora del desarrollo integral de la persona humana, que, mediante la caridad y la solidaridad, contribuye a la construcción de una sociedad más justa y fraterna, constituida por personas protagonistas de su historia.

\section{Proyecto "Adopción del Colegio Politécnico Alejandro Taboada"-Petroperú}

En el marco de la movilización nacional por la educación, del Ministerio de Educación del Perú, la empresa Petroperú Operaciones Talara adopta 
a la Institución Educativa (I.E.) Politécnico "Alejandro Taboada", ubicada en avenida Cementerio s/n, Asentamiento Humano San Pedro (Cono Norte) del distrito de Pariñas, provincia de Talara. Este colegio politécnico es una institución educativa de nivel secundaria con mil alumnos, que ofrece, además de la educación regular, una formación educativa industrial en las especialidades de mecánica de producción, mecánica automotriz, electricidad y electrónica. Asimismo, es importante señalar que el $84,2 \%$ de los escolares pertenece al estrato social bajo, y el 36,8 \% se dedica, además de estudiar, a trabajar en la pesca o en otros sectores.

El proyecto para su ejecución realizó alianzas estratégicas con la Unidad de Gestión Educativa Local (Ugel Talara), Senati y Sencico; las dos últimas, institutos técnicos de mecánica automotriz, soldadura y otros.

\subsection{Objetivos del proyecto}

Contribuir al desarrollo educativo, cultural, social, económico y del medio ambiente de la comunidad de Talara. Mejorar la formación regular y técnica de los escolares del politécnico y disminuir el número de egresados sin estudios ni empleo. Generar beneficios mutuos en las interacciones y acuerdos con los grupos de interés de nuestro entorno.

\subsection{Componentes del proyecto}

La adopción contribuye a la mejora de la formación regular y técnica de los alumnos, así como a su calidad de vida, mediante el apoyo progresivo de la empresa en aspectos de servicios (educativos, salud y medio ambiente), equipamiento e infraestructura, y a través del desarrollo de las siguientes actividades:

- Capacitación continua a los estudiantes con el dictado de clases de matemáticas, física, química, técnicas de soldadura y torno, con profesionales y técnicos voluntarios de la refinería Talara.

- Capacitación al personal docente y administrativo, a través de charlas, talleres y cursos dictados por profesionales de la refinería Talara y externos.

- Implementación de los talleres con materiales, herramientas y equipos necesarios para la realización de las prácticas de los alumnos.

- Construcción de los servicios higiénicos en las instalaciones del colegio politécnico. 
Así mismo, fue importante el involucramiento del personal de la empresa a través del programa de voluntariado, en el cual se compromete a los trabajadores de la empresa con la realización de actividades que les permitan contribuir al desarrollo de la comunidad, como el dictado de clases a los alumnos y otras.

\subsection{Impacto del proyecto}

El proyecto sigue vigente a la fecha y hasta el momento ha tenido un impacto en la mejora de la calidad de la educación de más de mil estudiantes de nivel secundaria, 60 docentes de la I.E. Politécnico "Alejandro Taboada" y 15 auxiliares y personal administrativo. En suma, 1085 personas beneficiadas directamente y 1085 familias, indirectamente, haciendo un total aproximado de 4340 personas.

Los alumnos, gracias al programa, fortalecieron sus capacidades, siguieron estudios superiores, preferentemente técnicos, e incrementaron sus posibilidades de inserción laboral. De esta manera, el proyecto logró el aumento del porcentaje de alumnos egresados que se encuentran estudiando una carrera técnica o profesional, y también disminuyó el porcentaje de alumnos egresados que se encuentran desempleados o sin estudiar una carrera técnica o profesional. Por otro lado, la empresa logró mejorar sus relaciones de aceptación, comprensión y apoyo mutuo de operaciones en Talara con sus grupos de interés.

\section{Programa "Leer es estar adelante"}

Este programa es una iniciativa de la Fundación BBVA-Continental del Banco BBVA-Continental, dentro de sus políticas de responsabilidad y siguiendo su enfoque de la promoción de la educación y la cultura. El programa cuenta con el respaldo del Ministerio de Educación y las unidades de gestión educativa (Ugel), y tiene como socio técnico al Instituto de Estudios Peruanos (IEP), quien diseñó la metodología para la ejecución del proyecto en las instituciones educativas estatales.

El programa se inició en el 2007 con un grupo de 19 escuelas localizadas en cinco regiones del Perú: Arequipa, Ayacucho, Lima, Loreto y Piura. Entre los años 2008 y 2010 se realizó una medición longitudinal del logro de aprendizaje a través de tres pruebas a los alumnos. Y desde el 2010 hasta la actualidad, el programa ha incorporado a dos nuevas regiones: Ancash y La Libertad. En el 2011, se unió una nueva región, Ica. 


\subsection{Objetivo del proyecto}

Elevar los niveles de comprensión lectora de los estudiantes de escuelas públicas de tercero, cuarto, quinto y sexto grados de primaria. El proyecto surge del interés y compromiso del banco por contribuir a la mejora de la calidad de la educación peruana a través de un esfuerzo que incluya a los estudiantes, familias, maestros y escuelas. Con el proyecto, se está ayudando a fortalecer capacidades en los futuros ciudadanos y posibles colaboradores de la empresa.

\subsection{Componentes del programa}

- Material educativo: Constituye el elemento central del programa, y consiste en la elaboración de textos para los alumnos y guías pedagógicas para los profesores, de acuerdo con la zona de intervención del programa, es decir, para cada región se desarrolla un libro específico. El libro se estructura en dos partes. En ambas se trabajan las habilidades y estrategias en tres momentos: antes de la lectura, durante la lectura y después de la lectura. Los libros siguen una línea temática que se organiza alrededor de la diversidad peruana, ya sea biológica, geográfica, cultural o étnica. Para reforzar dicha idea se han creado personajes característicos de cada lugar, lo cual es muy apreciado por los alumnos, según nuestra investigación.

- Capacitación docente: Este componente fortalece en los maestros un conjunto de habilidades conceptuales, pedagógicas y metodológicas para la enseñanza de la lectura comprensiva. Los maestros, además, son acompañados en su proceso de formación y reciben retroalimentación constante sobre su desempeño.

\subsection{Impacto del proyecto}

El programa "Leer es estar adelante", que sigue vigente hasta la fecha, ha beneficiado a más de noventa mil escolares de ocho regiones del país, en un total de 935 escuelas. Este crecimiento ha sido posible gracias a las alianzas sólidas con entidades públicas y privadas en los últimos tres años, lo cual ha permitido multiplicar el nivel de impacto del proyecto.

Las cifras de mejora en la comprensión lectora en los niños beneficiados han revertido las encontradas al inicio del proyecto. Según los indicadores elaborados por el IEP, solamente tres de cada diez niños 
comprendían lo que leían. En la actualidad, en los colegios intervenidos, la cifra señala que siete de cada diez niños comprenden lo que leen.

\section{Proyecto "Ally Micuy"-Cáritas del Perú y minera Antamina}

Este proyecto fue desarrollado entre septiembre del año 2007 y noviembre del año 2010, con el financiamiento del Fondo Minero Antamina. Cáritas del Perú trabajó en once de las veinte provincias de la región Ancash, en la zona norte de la región. El contexto de este proyecto nace a finales del año 2006, cuando en el Perú, gracias al acuerdo entre el gobierno peruano y las empresas mineras más grandes, existía el programa minero de solidaridad con el pueblo, denominado "Fondo Minero", compuesto por un aporte económico voluntario, extraordinario y temporal de las empresas mineras, que por aquel entonces tenían ganancias extraordinarias por el incremento del precio internacional de los minerales.

En este marco, la minera Antamina, asociada a las ONG Cáritas y ADRA, diseñó el proyecto de reducción de la desnutrición crónica infantil en comunidades de la región Ancash, denominado, en forma breve, "Ally Micuy", frase quechua que significa "buen alimento" o "alimentarse bien". Luego de varios meses de gestiones tripartitas entre la Asociación Antamina -entidad creada para la administración del Fondo Minero Antamina (FMA)-, ADRA y Cáritas del Perú, en el mes de agosto del 2007 se firmó el convenio bilateral entre el FMA y Cáritas del Perú.

\subsection{Objetivos del proyecto}

Contribuir al desarrollo humano sostenible de comunidades pobres de las once provincias de la región Ancash, mejorando el estado de salud y nutrición de los niños menores de tres años; teniendo como meta disminuir la prevalencia de desnutrición crónica en niños y niñas menores de tres años, en siete puntos porcentuales, y de anemia por deficiencia de hierro, en diez puntos porcentuales.

\subsection{Componentes del proyecto}

El proyecto "Ally Micuy" desarrolló una estrategia basada en tres componentes: mejorar la alimentación y cuidado de los menores, mejorar las condiciones en la vivienda donde habita el menor de edad, y aumentar el involucramiento de las autoridades, para dar sostenibilidad al proyecto. 
- Prácticas en alimentación, cuidado y atención de salud: Este componente estuvo basado en la educación comunitaria en salud y nutrición, como un pilar fundamental para el cambio de prácticas familiares orientadas a mejorar la alimentación, la atención y el cuidado del niño y niña.

- Implementación progresiva de la estrategia de "vivienda rural saludable": Debido a que la condición sanitaria de la vivienda es un factor determinante para la salud y nutrición humanas, era importante ayudar a las familias a tener una vivienda saludable. Dentro de ello, el componente higiene es vital para la preparación de alimentos, la forma en que se cocinan y cómo se manipulan en el hogar.

- Involucramiento de autoridades comunales y municipales: Este proyecto, buscando asegurar la sostenibilidad social y económica de los resultados de la intervención, desarrolló un conjunto de acciones para comprometer a los alcaldes locales a participar activamente en él, priorizando la inversión en salud y nutrición como una estrategia de superación de la pobreza.

\subsection{Impacto del proyecto}

En los tres años que duró, el proyecto logró los objetivos propuestos, y consiguió una disminución importante de la prevalencia de la desnutrición crónica en los niños menores de tres años, del $35 \%$ al $28 \%$. Además, se demostró que la siempre supervisada administración del sulfato ferroso es efectiva: dos veces por semana, disminuye la anemia en niños entre 6 y 35 meses de edad.

\section{Condiciones para la puesta en marcha de la gestión de responsabilidad social en las organizaciones}

La siguiente matriz describe los tres tipos de áreas que deben cubrirse dentro de la gestión de acciones de responsabilidad social empresarial en una organización. El cuadro siguiente describe tres columnas: la primera, compuesta por el ámbito corporativo, en el cual se señala una serie de exigencias que la empresa u organización debe tener en cuenta para la gestión de políticas y prácticas de responsabilidad social; la segunda columna está compuesta por aspectos que deben contener los proyectos, programas o acciones; y finalmente, la tercera columna hace 
referencia a la medición del impacto de las acciones de responsabilidad social con todos los grupos de interés.

Cuadro 1.

Condiciones que deben tener las empresas para la gestión de proyectos y prácticas de responsabilidad social

\begin{tabular}{|c|c|c|}
\hline $\begin{array}{l}\text { Condiciones de la } \\
\text { organización }\end{array}$ & $\begin{array}{l}\text { Condiciones de los } \\
\text { programas y proyectos }\end{array}$ & $\begin{array}{l}\text { Medición del impacto de las } \\
\text { acciones }\end{array}$ \\
\hline $\begin{array}{l}\text { Alineamiento de las políticas } \\
\text { de responsabilidad social con } \\
\text { la misión, visión, principios de } \\
\text { la empresa u organización. }\end{array}$ & $\begin{array}{l}\text { Larga duración del proyecto } \\
\text { (más de tres años permanen- } \\
\text { tes, a renovarse por periodos } \\
\text { de cinco a diez años). }\end{array}$ & $\begin{array}{l}\text { Presencia de indicadores de medi- } \\
\text { ción y eficacia del proyecto a diverso } \\
\text { nivel: de participación, de satisfac- } \\
\text { ción de parte de los beneficiarios, etc. }\end{array}$ \\
\hline $\begin{array}{l}\text { Compromiso de la alta } \\
\text { dirección, directivos y } \\
\text { gerentes de la empresa con } \\
\text { las políticas y prácticas de } \\
\text { responsabilidad social. }\end{array}$ & $\begin{array}{l}\text { Alianzas con otras organiza- } \\
\text { ciones a nivel técnico y econó- } \\
\text { mico, para la ejecución de } \\
\text { programas y proyectos. }\end{array}$ & $\begin{array}{l}\text { Impacto positivo en los beneficia- } \\
\text { rios. Reconocimiento de parte de } \\
\text { los beneficiarios. }\end{array}$ \\
\hline
\end{tabular}

\begin{tabular}{lll}
\hline Conocimiento de las políti- & Compromiso de los trabajado- & Impacto positivo en la imagen y \\
cas y prácticas de respon- & res involucrados en el proyec- & reputación de la empresa u organi- \\
sabilidad social por parte de & to: equipo técnico-ejecutor. & zación, por parte de los diversos \\
los colaboradores y trabaja- & & grupos de interés.
\end{tabular}
dores del proyecto.

Compromiso y motivación de los colaboradores que trabajan para el proyecto/ programa.

Presencia de materiales y herramientas de comunicación como componentes que ayuden a ejecutar el proyecto/programa y a lograr su mejor llegada a los diversos grupos de interés.

Buena cultura corporativa Comunicación del éxito del en el desarrollo del proyecto. proyecto a los beneficiarios y a los grupos de interés internos y externos de la empresa.

Alineamiento del proyecto/programa con las políticas públicas actuales del Gobierno. Esto ayudará a buscar la sostenibilidad del proyecto. dar a conocer las acciones de responsabilidad social de la empresa u organización.
Capacidad de autosostenimiento del proyecto a futuro.

Alianzas con las autoridades, comunidades, organizaciones del tercer sector e inclusive otras empresas con quienes se relacione o se pueda relacionar el proyecto.

Involucramiento de la comunidad y empoderamiento de los beneficiarios del proyecto.

Fuente: Elaboración propia 


\section{Indicadores para la medición de la eficacia de la sostenibilidad de los proyectos de responsabilidad social}

Uno de los principales desafíos que tienen los proyectos sociales es la sostenibilidad. En los tres casos analizados hemos evidenciado que el mayor problema está enfocado en encontrar la manera más efectiva de lograr el compromiso de los actores internos y externos con el proyecto, para lograr la multiplicación, mejora y continuidad de este. Una de las rutas es el involucramiento de las autoridades locales y los trabajadores de las organizaciones públicas, sobre todo de los sectores de educación y salud. Sin embargo, esto resulta un tanto débil debido a la permanente rotación de personal y al cambio de autoridades. Por otra parte, es importante trabajar en el empoderamiento, fortalecimiento y la capacidad de gestión de los propios beneficiarios, lo cual termina siendo una labor mucho más complicada debido a múltiples factores, pero que podría mejorar la efectividad. También se pueden pensar en alianzas con otras organizaciones de la sociedad civil, como las ONG, asociaciones sin fines de lucro, e inclusive empresas. Esta fórmula puede resultar innovadora y eficaz.

Sin embargo, frente a diversos contextos en los que se ejecutan los programas sociales, es necesario orientar los esfuerzos para no solo pensar en el presente sino también en el futuro. Pues el camino para el desarrollo social y las mejoras de las condiciones de vida de las poblaciones es un trabajo permanente, donde deben laborar todos los sectores de la sociedad, empresas, sociedad civil y gobierno. Cada proyecto es un caso diferente; por ende, no existen recetas de efectividad para los proyectos, pero sí algunas variables que se deben tener en cuenta para su ejecución.

A continuación presentamos algunos indicadores a tener en cuenta al momento de gestionar un programa o proyecto social desde un enfoque de responsabilidad social. 
Cuadro 2.

Indicadores para la efectividad de los proyectos de responsabilidad social

\begin{tabular}{cl}
\hline Variables & Indicadores \\
\hline A nivel empresarial u organizacional & \\
\hline
\end{tabular}

Alineamiento del proyecto con la misión, Número de proyectos relacionados con principios y visión, los principios y el concepto de conceptos de RS de la empresa.

responsabilidad social de la empresa.

Compromiso de la alta dirección y los Número de directores que están comprometidos con gerentes de la empresa. el proyecto/programa.

Número de gerentes y directivos comprometidos con el proyecto/programa.

Conocimiento del proyecto por parte de Número de trabajadores y colaboradores que están los colaboradores y trabajadores. informados sobre el proyecto.

Número de trabajadores y colaboradores que han visitado 0 son voluntarios del proyecto.

Buena cultura corporativa de la empresa. Número de trabajadores y colaboradores que se identifican con el proyecto.

\section{A nivel del proyecto/programa}

Larga duración del proyecto (más de tres Número de años que se ejecuta el proyecto. años permanentes).

Alianzas con otras organizaciones a nivel Número de alianzas realizadas con empresas e institécnico y económico, para la ejecución tuciones en el proyecto.

del proyecto en la comunidad.

Compromiso de las personas involucra- Número de trabajadores comprometidos con el prodas en el proyecto: equipo técnico-ejecu- yecto (nivel de compromiso).

tor.

Presencia de indicadores de medición y eficacia del proyecto.

Número de variables con las cuales medir la eficacia del proyecto en los beneficiarios.

Capacidad de autosostenimiento del proyecto a futuro.

Número de posibilidades de ser sostenible que tiene el proyecto.

Alineamiento del proyecto/programa con políticas públicas actuales.

Nivel de impacto del proyecto con respecto a las politicas públicas actuales del Gobierno.

Presencia de materiales que ayuden a Número de materiales realizados para el proyecto. ejecutar el proyecto/programa.

Impacto positivo en los beneficiarios. Número de beneficiarios.

Nivel de impacto.

Impacto positivo en la empresa. Número de vinculaciones del proyecto con ranking de imagen o reputación corporativa de la empresa. 
continuación

\begin{tabular}{ll}
\hline \multicolumn{1}{c}{ A nivel de comunicación } & \\
\hline $\begin{array}{l}\text { Comunicación del éxito del proyecto a } \\
\text { los beneficiarios y stakeholders internos. }\end{array}$ & $\begin{array}{l}\text { Número de publicaciones realizadas acerca } \\
\text { del proyecto. } \\
\text { Nivel de conocimiento del proyecto. }\end{array}$ \\
$\begin{array}{ll}\text { Comunicación del éxito del proyecto a } \\
\text { los beneficiarios y stakeholders externos. }\end{array}$ & $\begin{array}{l}\text { Número de publicaciones realizadas acerca } \\
\text { del proyecto. } \\
\text { Nivel de conocimiento. }\end{array}$ \\
$\begin{array}{ll}\text { Relaciones públicas con los medios de } \\
\text { comunicación. }\end{array}$ & $\begin{array}{l}\text { Número de medios de comunicación que conocen } \\
\text { del proyecto. } \\
\text { Número de publicaciones en medios de } \\
\text { comunicación. }\end{array}$ \\
$\begin{array}{ll}\text { Materiales de comunicación y difusión } \\
\text { realizados sobre el proyecto. }\end{array}$ & $\begin{array}{l}\text { Número de materiales realizados. } \\
\text { Elaboración del reporte de sostenibilidad } \\
\text { social o balance social. }\end{array}$ \\
\hline
\end{tabular}

Fuente: Elaboración propia

\section{Conclusiones}

El ejercicio de la responsabilidad social en las empresas y organizaciones debe nacer de la voluntad de las propias empresas, lo cual se debe manifestar en la misión, visión, principios y valores. La responsabilidad es un acto voluntario que debe buscar un modelo de gestión único y siempre persiguiendo las buenas relaciones con el entorno, es decir, con los diversos grupos de interés de la organización.

Los programas sociales pueden ser acciones muy efectivas para la gestión de responsabilidad social de una empresa pública, privada o del tercer sector, sin importar el tamaño, rubro o modelo de gestión económico de la organización.

Las acciones de responsabilidad social más representativas en el país son los temas vinculados a la educación, salud, el medio ambiente, desarrollo de capacidades, etc., temas que tienen un impacto importante debido al cambio social que pueden representar en la sociedad. Esto además tiene un doble impacto, puesto que también representa un valor para la imagen y la reputación de la empresa u organización.

Las empresas necesitan alianzas técnicas, económicas, académicas y otras, con las organizaciones de diversos sectores - públicas, privadas y 
del tercer sector-, para la ejecución de variados programas y proyectos, dentro de una lógica de colaboración y un "ganar-ganar" compartido.

Los programas sociales son las acciones más visibles de las empresas. En este sentido, traerán buenos resultados para la imagen y la reputación en las organizaciones. Sin embargo, siempre hay que tener claro que la imagen y la reputación deben entenderse como un resultado pero no como un objetivo; de lo contrario, el impacto podría ser negativo en la imagen de la propia organización.

Uno de los retos más importantes de la gestión de las políticas de responsabilidad social es el logro de la sostenibilidad de los programas y proyectos en el tiempo. Para superar estos inconvenientes, las organizaciones o empresas deben realizar acciones que cumplan con la premisa del "ganar-ganar". Es decir, la acción debe traer beneficios que impacten positivamente en los grupos de interés de la organización, y también para la propia empresa.

La comunicación es una herramienta clave en el proceso de puesta en marcha de la responsabilidad social en la organización, debido a que ayuda a conocer y mantener el diálogo con los grupos de interés. La responsabilidad social exige un mapeo, conocimiento y diálogo permanente con todos los grupos de interés.

\section{Referencias}

BBVA-Continental. Informe anual 2011. (Abril-mayo del 2012). <http:// www. bbvabancocontinental.com.pe/> [Fecha de consulta, 12 de mayo del 2013.]

Cáritas del Perú. Balance social 2011. (Octubre del 2012). <http://www. caritas.org.pe/> [Fecha de consulta, 14 de mayo del 2013.]

García, J. (2004). Las cuentas de la economía social: el tercer sector en España. Madrid: Civitas Ediciones.

Navarro, F. (2008). Responsabilidad social corporativa: teoría y práctica. Madrid: ESIC.

Petroperú. Memoria social 2011. (Julio del 2012). <http://www.petroperu.com.pe/> [Fecha de consulta, 10 de mayo del 2013.]

Salas, L. (2004). Razón de ser de las organizaciones no gubernamentales. Fundamentos, 20. Bogotá: Fundación Saldarriaga Concha, 19.

Schwalb, M., \& Malca, O. (2005). Responsabilidad social: fundamentos para la competitividad empresarial y el desarrollo sostenible. Lima: CIUP. 


\section{Anexos}

Anexo 1.

Listado de personas entrevistadas en Petroperú-Lima.

\begin{tabular}{ll}
\hline \multicolumn{1}{c}{ CARGO } & \multicolumn{1}{c}{ NOMBRE } \\
\hline Presidente del directorio & Ing. Héctor Reyes \\
Gerente del Departamento de Desarrollo Sostenible & Javier Alegría Tenorio \\
Jefa de la Unidad de Gestión Social & Clara Indacochea Álvarez \\
Jefe de la Unidad de Gestión Ambiental & Juan Gallarday Pretto \\
Jefa del Área de Imagen & Carla Santacruz \\
Jefe de la Unidad de Gestión Cultural & Carlos del Águila \\
Jefe del Área de Medios & Juan José Beteta \\
Jefe de la Unidad de Relaciones Industriales & César Suazo \\
Supervisora de Responsabilidad Social-Oleoducto & Raquel Vilela \\
Supervisor de Responsabilidad Social-Refinería Conchán & Julio Guillermo Estrada Basurto \\
Supervisora de Responsabilidad Social-Refinería Selva & Sintya Vilca Villalobos \\
Supervisor de la Unidad de Gestión Ambiental & Alfredo Pinillos Cáceres \\
Supervisora de proyectos de la Unidad de & María Teresa Gonzales \\
Gestión Cultural y Proyectos Sociales & \\
Supervisora de Bienestar & Guelly Álvarez \\
Supervisora de Proyectos & Milagros Bermeo \\
Supervisora de Bienestar & Silvana Gambini \\
Supervisora de Salud Ocupacional & Karin Alkon \\
Supervisora del Museo del Petróleo & Xenia Poblet \\
\hline
\end{tabular}

Anexo 2.

Listado de personas entrevistadas de BBVA-Continental-Lima.

\begin{tabular}{ll}
\hline \multicolumn{1}{c}{ CARGO } & \multicolumn{1}{c}{ NOMBRE } \\
\hline Gerente general & Eduardo Torres-Llosa \\
Director de Comunicaciones y de la Fundación & Carlo Reyes \\
BBVA-Continental & \\
Coordinadora de Relaciones Externas & Mercedes Castro \\
\hline
\end{tabular}


Anexo 3.

Listado de personas entrevistadas de Cáritas del Perú-Lima.

\begin{tabular}{ll}
\hline \multicolumn{1}{c}{ CARGO } & \multicolumn{1}{c}{ NOMBRE } \\
\hline Presidente de Cáritas & Mons. Richard Alarcón \\
Secretario general & Jorge Lafosse \\
Gerente de Gestión y Administración de Recursos & Manuel Heredia G. \\
Gerente de Desarrollo Social & Andrés Moran Tello \\
Gerente de Desarrollo de Proyectos & Héctor Hanashiro \\
Gerente de Desarrollo Económico & Juan Pablo Silva Castañeda \\
Asesor pastoral & Mons. Hugo Garaycoa \\
Responsable de la Unidad de Asesoría Pastoral & Roberto Tarazona \\
Responsable de la Unidad de Imagen Institucional & Carla Ausa \\
Asesora legal & Patricia Otero Ramírez \\
Asistente de soporte informático & Sonia Leiva \\
\hline
\end{tabular}

Anexo 4.

Listado de personas entrevistadas del proyecto "Adopción del Colegio Politécnico Alejandro Taboada"-Talara, Piura, de la empresa Petroperú.

\begin{tabular}{ll}
\hline \multicolumn{1}{c}{ CARGo } & \multicolumn{1}{c}{ NOMBRE } \\
\hline Gerente de Petroperú-Talara & José Hidalgo \\
\hline Jefa de Relaciones Comunitarias-Talara & Griselda López \\
Supervisor de Responsabilidad Social-Talara & Gonzalo Gonzales \\
Auxiliar de Responsabilidad Social-Talara & Darío Ruiz \\
Supervisora de Bienestar Social-Talara & Beatriz Ojeda \\
Supervisor de Comunicación Externa-Talara & Carlos Vega \\
Director de la Ugel-Talara-Piura & Luis Meza Velásquez \\
Director del Colegio Politécnico Alejandro Taboada & Eduardo Panta Zapata \\
Docente del Colegio Politécnico Alejandro Taboada & Carmen Santillán More \\
Docente del Colegio Politécnico Alejandro Taboada & Esmelda Vera Zapata \\
Docente técnico del Colegio Politécnico Alejandro Taboada & Jorge Albordoz Zevallos \\
Presidente de la Apafa del Politécnico Alejandro Taboada & Reymundo Coronado Cáceres \\
Alumno del Colegio Politécnico Alejandro Taboada & Erick Talledo Cubos \\
Alumno del Colegio Politécnico Alejandro Taboada & Kevin Marcelo Barrientes \\
Alumna del Colegio Politécnico Alejandro Taboada & Mirella Morán Rojas \\
Ex alumno del Colegio Politécnico Alejandro Taboada & Edwin López Panta \\
Ex alumno del Colegio Politécnico Alejandro Taboada & Juan Sandoval Peña \\
Ex alumna del Colegio Politécnico Alejandro Taboada & Lucero Loro Payva \\
Padre de familia del Colegio Politécnico Alejandro Taboada & Hernán Alvarado Calero \\
\hline
\end{tabular}


Anexo 5.

Listado de personas entrevistadas del Programa "Leer es estar adelante", de la empresa BBVA-Continental-Lima.

\begin{tabular}{ll}
\hline \multicolumn{1}{c}{ CARGO } & \multicolumn{1}{c}{ NOMBRE } \\
\hline Coordinador del IEP & Dynnick Ascencios \\
Coordinadora del IEP & Mariana Eguren \\
Coordinadora general del proyecto del IEP & Natalia González \\
Coordinador de colegios IEP & Luis Peralta \\
Directora del Colegio Javier Pérez de Cuéllar & Iraida Araujo \\
Subdirector del Colegio Javier Pérez de Cuéllar & Tito Pérez \\
Docente del Colegio Javier Pérez de Cuéllar & Lindomira Vásquez \\
Docente del Colegio Javier Pérez de Cuéllar & Mercedes Arrieta \\
Alumno del Colegio Javier Pérez de Cuéllar & Piero Véliz \\
Alumna del Colegio Javier Pérez de Cuéllar & Mabel Castro \\
Madre de familia del Colegio Javier Pérez de Cuéllar & Mariana Eguren \\
Madre de familia del Colegio Javier Pérez de Cuéllar & Sobeida Mena \\
\hline
\end{tabular}

\section{Anexo 6.}

Listado de personas entrevistadas del proyecto "Ally Micuy", de Cáritas del Perú y Antamina. Distrito de Cashapampa-Provincia de Siguas-Región Ancash.

\begin{tabular}{ll}
\hline \multicolumn{1}{c}{ CARGO } & \multicolumn{1}{c}{ NOMBRE } \\
\hline Alcalde de la municipalidad de Cashapampa & Uldarico Cisneros \\
Gerente de la municipalidad de Cashapampa & Ernesto Padilla \\
Oficina de Desarrollo Social Cashapampa & Sandra Morilla \\
Coordinador general del proyecto Ally Micuy & Javier Garay \\
Coordinador distrital del proyecto Ally Micuy & Percy Vilcarano \\
Doctor cerum de la posta de Cashapampa & Luis Enrique Mejía Ruiz \\
Doctora cerum de la posta de Cashapampa & María Paydanpan \\
Enfermera de la posta de Cashapampa & Marina Norabuena \\
Enfermera técnica de la posta de Cashapampa & Nataly Rojas \\
Promotor de salud y educador & Wálter Castillo \\
Educadora del proyecto Ally Micuy & Éricka Villanueva \\
Educadora del proyecto Ally Micuy & Alicia Padilla \\
Educadora del proyecto Ally Micuy & Reyna Zabino \\
Madre beneficiaria del proyecto Ally Micuy & Lorza Vergaray \\
Madre beneficiaria del proyecto Ally Micuy & María Mili Santisteban \\
Locutora de Radio Corazón de Cashapampa & Nila Bermúdez \\
Locutor de Radio Corazón de Cashapampa & Wiliam Montero \\
\hline
\end{tabular}

